\title{
Polarity Establishment and Maintenance in Ascidian Notochord
}

\author{
Hongzhe Peng ${ }^{1}$, Runyu Qiao ${ }^{1}$ and Bo Dong 1,2,3* \\ ${ }^{1}$ Sars-Fang Centre, MoE Key Laboratory of Marine Genetics and Breeding, College of Marine Life Sciences, Ocean \\ University of China, Qingdao, China, ${ }^{2}$ Laboratory for Marine Biology and Biotechnology, Qingdao National Laboratory \\ for Marine Science and Technology, Qingdao, China, ${ }^{3}$ Institute of Evolution and Marine Biodiversity, Ocean University of \\ China, Qingdao, China
}

OPEN ACCESS

Edited by:

Yi Wu,

UConn Health, United States

Reviewed by:

Ana Carmena

Consejo Superior de Investigaciones Científicas (CSIC), Spain

Sally Ann Moody,

George Washington University,

United States

*Correspondence:

Bo Dong

bodong@ouc.edu.cn

Specialty section:

This article was submitted to

Signaling,

a section of the journal

Frontiers in Cell and Developmental

Biology

Received: 21 August 2020 Accepted: 12 October 2020 Published: 30 October 2020

Citation:

Peng H, Qiao R and Dong B (2020) Polarity Establishment and Maintenance in Ascidian

Notochord.

Front. Cell Dev. Biol. 8:597446. doi: 10.3389/fcell.2020.597446
Cell and tissue polarity due to the extracellular signaling and intracellular gene cascades, in turn, signals the directed cell behaviors and asymmetric tissue architectures that play a crucial role in organogenesis and embryogenesis. The notochord is a characteristic midline organ in chordate embryos that supports the body structure and produces positioning signaling. This review summarizes cellular and tissue-level polarities during notochord development in ascidians. At the early stage, planar cell polarity (PCP) is initialized, which drives cell convergence extension and migration to form a rod-like structure. Subsequently, the notochord undergoes a mesenchymal-epithelial transition, becoming an unusual epithelium in which cells have two opposing apical domains facing the extracellular lumen deposited between adjacent notochord cells controlled by apical-basal (AB) polarity. Cytoskeleton distribution is one of the main downstream events of cell polarity. Some cytoskeleton polarity patterns are a consequence of PCP: however, an additional polarized cytoskeleton, together with Rho signaling, might serve as a guide for correct AB polarity initiation in the notochord. In addition, the notochord's mechanical properties are associated with polarity establishment and transformation, which bridge signaling regulation and tissue mechanical properties that enable the coordinated organogenesis during embryo development.

Keywords: notochord, planar cell polarity, apical-basal polarity, initiation of $A B$ polarity, lumen formation

\section{INTRODUCTION}

Cell proliferation, migration, and deformation; extracellular matrix (ECM) secretion; and cellcell junction formation and remodeling are basic processes in tissue and organ architecture establishment during embryogenesis. However, these processes are not always isotropic, and anisotropic processes lead to spatial differences in cell shape and structure, directed cell behaviors (e.g., migration, mitosis, adhesion, secretion, and signaling transition), and asymmetric subcellular structures (e.g., organelle localization and cytoskeleton distribution) all of which generate what is called "polarity" (Dhonukshe, 2013; Wolpert, 2013). Polarity widely exists during the entire lifespan and at all levels of an organism. At the tissue level, polarity appears as asymmetric differentiation forming distinct cell types and/or the mechanically anisotropic tissues. For the whole embryo, polarity manifests as the formation of orientated body axis and the asymmetric location of organs.

Polarity is regulated by signaling pathways that respond to chemical stimulations such as morphogens (Turing, 1952; Pickett et al., 2019) or physical stimulation like light 
(Brownlee and Bouget, 1998) from the extracellular space, where oriented signals are sent, except a small part of spontaneous random cell polarization (Wedlich-Soldner and Li, 2003). Extracellular signals transmit through paracrine pathways mediated by morphogen concentration gradient signaling, such as Wnt signaling (Ukita et al., 2009), Notch signaling (Hermann et al., 2000; Kato, 2011), Hedgehog signaling (Choi and Harfe, 2011; Hu et al., 2017), and transforming growth factor beta signaling (bone morphogenetic protein, activin) (Ninomiya, 2004; Eom et al., 2011). In addition, cells also receive orientation signals through contacts, such as cell-cell adhesion (Nance, 2014) or cell-ECM network like basement membrane adhesion (Marsden and DeSimone, 2003). These extracellular signals provide directional information, which induces asymmetric distribution of protein complexes or subcellular organelles, establishing intracellular polarity (Pickett et al., 2019). There are two main intracellular polarity signal systems, apical-basal (AB) polarity mediated by the Par/atypical protein kinase C (aPKC) complex (Assémat et al., 2008), and planar cell polarity $(\mathrm{PCP})$, mediated by Frizzled (Fz, also called Fzd in vertebrate)- Flamingo (Fmi, also known as starry night in Drosophila and Celsr in vertebrate)- Disheveled (Dsh, also called Dvl in vertebrate)- Diego (Dgo, also called Diversin or Inversin in vertebrate), and Van Gogh (Vang, also known as strabismus in Drosophila and Vangl in vertebrate)- Flamingo (Fmi)- Prickle (Pk, also known as prickle-spiny legs in Drosophila) complexes (Adler, 2012; Yang and Mlodzik, 2015). Both intracellular polarity signal systems coexist in cells and sometimes cooperate too (Djiane et al., 2005; Wu and Mlodzik, 2009), contributing to the formation and maintenance of multidimensional polarity.

Polarity establishment is necessary for morphogenesis, by which three-dimensional (3D) complex architectures emerge from symmetric, simple structures. Tissue and organ specialization and cell fate depend on the direction and position information. For example, anterior-posterior (AP) axis formation regulates somite differentiation (Bénazéraf and Pourquié, 2013), while left-right (LR) axis formation regulates the asymmetry location and differentiation of the brain, heart, and gut (Bisgrove et al., 2000; Hashimoto and Hamada, 2010; Grimes, 2019). The body plan symmetry of animals is diversified including spherical symmetry, radial symmetry, biradial symmetry, and bilateral symmetry (Holló, 2015). In bilaterally symmetric animals, some inner organs, such as the heart and gut, in the symmetric body plan show LR asymmetry (Raya and Belmonte, 2006).

Of multi-leveled polarities, tissue polarity, formed from cell polarity (Mlodzik, 2002) and manifesting as tissue mechanical property and tissue movement, epitomizes the polarity phenomena and plays an important role in developmental processes.

\section{THE NOTOCHORD}

The notochord is a characteristic rod-like midline organ in chordate embryos that supports the body structure and produces signaling (Stemple, 2005; Corallo et al., 2015). Notochord evolution has three stages: muscle-like axochord (Lauri et al., 2014; Brunet et al., 2015), rigid cell cord, and osseous vertebral column. A notochord-like muscle structure has been reported in annelids (Lauri et al., 2014) indicating that the notochord originates from muscle (Lauri et al., 2014; Brunet et al., 2015). In hemichordatas, the lowest chordates, the stomochord, a notochord primordium, appears (Balser and Ruppert, 1990). In amphioxus, a cephalochordate, the notochord is present throughout the body during the entire lifespan as the body's main support structure (Feng et al., 2016). In urochordates, such as ascidians, the notochord only exists in the tail part in swimming larvae and disappears after metamorphosis (Cloney, 1982; Matsunobu and Sasakura, 2015). In cyclostomes, cartilaginous tissue appears around the notochord (Ota and Kuratani, 2007). In higher chordates, such as fish and mouse, the notochord exists centrally in the embryonic and larval body, and it is replaced by the spine in adults (Wopat et al., 2018; Bagwell et al., 2020). The notochord is converted into the nucleus pulposus in fully formed intervertebral discs, which protect the vertebrae from rubbing against each other (McCann et al., 2011).

\section{POLARITIES IN Ciona NOTOCHORD}

Ascidian is a basic group in chordate, with the notochord at larval stage. It is becoming an ideal model in marine invertebrates' morphogenesis (Lv et al., 2019). During notochord development in Ciona, diverse polarity patterns are built and play an important role in asymmetric structure formation and directional movement of notochord cells. First, notochord cells intercalate to form a rod-like structure along the midline of the body through convergence extension (CE) process regulated by a medial-lateral (ML)-oriented PCP (Figure 1A; Munro and Odell, 2002). During CE, ventrally polarized cytoskeleton contractility induces the notochord and the tail to bend ventrally and elongate posteriorly within the chorion (Figure 1B). Next, mesenchymalepithelial transition (MET) occurs, in which notochord cells become epithelial-like with AB polarity (Figures 1C,D). The PCP direction changes from ML to the one-dimensional (1D) AP axis (Kourakis et al., 2014). During the period, notochord cells secret the ECM into the basal, building a notochord sheath, and also into the apical surfaces, forming the extracellular lumen for cavitation (Figure 1E), which provides sufficient stiffness to support the body (Keller, 2006; Yasuoka, 2020). Polarity plays an essential role in all these processes. This review focuses on MET of notochord cells and polarity establishment and maintenance during this process.

\section{ESTABLISHMENT OF AB POLARITY IN Ciona NOTOCHORD}

A rod-like notochord appears after cell intercalation, in which PCP signaling sends polar signals parallel to the AP axis (Kourakis et al., 2014). The notochord elongates and undergoes MET, establishing AB polarity (Dong et al., 2009; Denker et al., 2013). The MET includes two main steps: (1) apical domains 
A intercalation

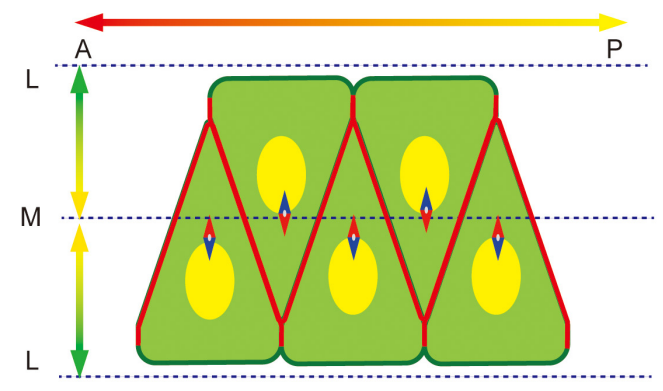

C

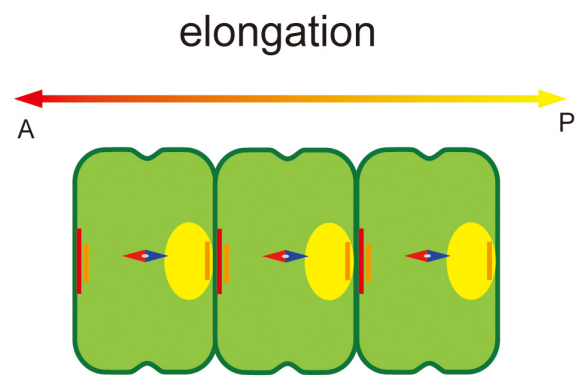

E

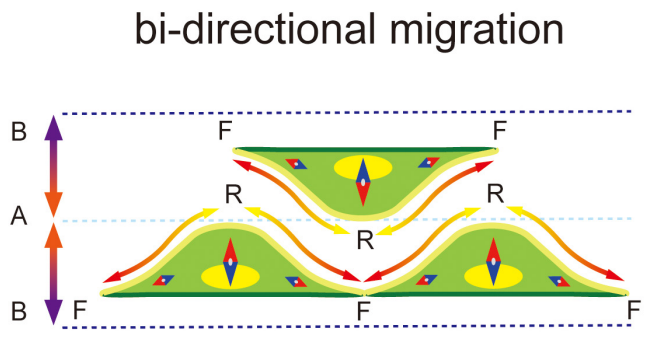

B

\section{bending}

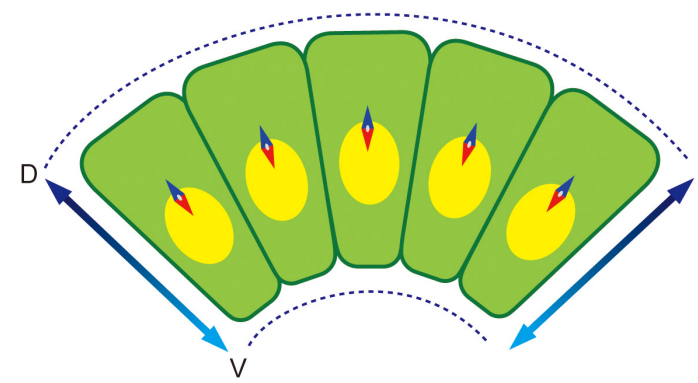

D

\section{lumen expansion}

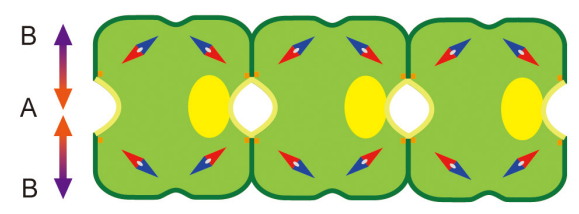

FIGURE 1 | Polarity at different notochord development stages in Ciona. (A) Notochord cells form a mediolateral (ML) polarity to drive the migration to the midline during convergence and extension. The notochord elongates parallel to the anterior-posterior (AP) axis regulated by embryonic AP polarity signaling. The key PCP component protein Prickle is located at the notochord cell-cell contact domain. (B) Notochord cells form a dorsal-ventral (DV) polarity to abend tissue toward the ventral side. (C) After cell intercalation, the PCP direction changes from ML to 1D AP axis. The Prickle relocates at the anterior edge of each notochord cell. (D) Apical-basal (AB) polarity is built to induces extracellular lumen formation. Two apical domains appear in one notochord cell. (E) Notochord cells migrate bidirectionally to induce the lumen connection. Adjacent notochord cells flatten opposite to each other along the notochord sheath.

initially appear at the center of two opposite lateral domains of each notochord cell; (2) extracellular lumen is deposited outside of apical domains and expanded continuedly (Denker and Jiang, 2012). The Par3-Par6-aPKC polarity complex is essential for $\mathrm{AB}$ polarity formation in epithelial cells (Assémat et al., 2008; Dhonukshe, 2013; Wen and Zhang, 2018). In Ciona notochord, Par3, Par6, and aPKC co-localize at the AP polar edges of notochord cells. Inhibition of Par3 function causes loss of the polarized localization of Par6 and aPKC, and the lumen cannot form, suggesting that Par3 might be the signaling upstream to guide $\mathrm{Par} / \mathrm{aPKC}$ and $\mathrm{AB}$ polarity formation. It has been known that knockdown of Par3 caused mislocalization of Par6 and aPKC, and blocked the $\mathrm{AB}$ polarity formation. However, mislocalization of Par6 using an aPKC binding dominant negative (PAR-6 $\triangle \mathrm{aPKC}-\mathrm{BD}$ ) (Suzuki et al., 2001) did not affect the localization of Par3. While, overexpression of myristoylated aPKC (myr-aPKC), which leads to the mislocalization of aPKC, caused the mislocalization of Par6. However, the localization of Par3 was unaffected (Denker et al., 2013). These results indicate that Par3 is upstream of aPKC and Par6, while the localization of Par6 depends on aPKC in Ciona notochord. The upstream signaling that induces the Par3 polar localization is still unknown. Some studies in Caenorhabditis elegans and Drosophila suggest that the 
localization of Par/aPKC complex is regulated by centrosome (Feldman and Priess, 2012). Engulfment and cell mobility (ELMO) family proteins might be involved in the regulatory process (Schmidt et al., 2018). With the development, ECM components are secreted out, forming the extracellular lumen at the anterior and posterior edges of notochord cells, and the notochord cell surface is separated into apical (facing the lumen), lateral (contact between neighboring cells), and basal membrane domains. After lumen formation, the Par3-Par6-aPKC polar complex localizes at the boundary between apical and lateral membranes to form a ring-like localization (Denker et al., 2013; Smith, 2018). Initially, each cell forms two apical domains in the notochord in Ciona, which then eventually merge into one (Figures 1D,E; Dong et al., 2009). During this process, notochord cells are regulated by polar signaling from both $\mathrm{PCP}$ and $\mathrm{AB}$ polarity that induces many proteins, such as cytoskeleton and cell junction proteins, toward polar localization. A contractile ring appears at the anterior side of each notochord cell and then moves to the equator to provide the force required for cell elongation (Dong et al., 2011). Zonula Occludens 1 (ZO1 ), as part of the tight junction, co-localizes with the Par3Par6-aPKC polar complex and they work together to occlude the lumen cavity (Denker et al., 2013). Solute Carrier 26a $\alpha$ (SLC26a $\alpha$ ), a transmembrane transport protein locates at the apical membrane to control the osmotic pressure in the lumen cavity (Deng et al., 2013).

The relationship between $\mathrm{PCP}$ and $\mathrm{AB}$ polarities, that is, whether and how they cross-talk, is still unclear. There exist three possible ways of communication: (1) Key PCP and AB polar proteins might interact directly. This type of cross-talk between PCP and AB polarities has been identified in the Drosophila eye, in which aPKC inhibits PCP activity by Fz1 phosphorylation (Djiane et al., 2005; Wu and Mlodzik, 2009). In Ciona notochord cell, Prickle and Par/aPKC complex are overlapped at the presumptive anterior apical domain (Jiang et al., 2005; Denker et al., 2013), offering the possibility for their direct interaction. (2) Notochord cells receive signals from neighboring cells. In intercalation abnormal mutant Ciona embryos, a notochord cell is usually in contact with more than two adjacent cells, and apical domains can be observed at each cell-cell contact (Denker et al., 2013), confirming that the AB polarity of notochord cells is affected by neighboring cells. When cell ablation removes the posterior neighboring cell, the notochord cell loses the posterior location of the nuclei. In addition, nucleus in the 40th notochord cell, which is the last cell in notochord tissue, locates in the anterior part of the cell (Kourakis et al., 2014). Both the observed facts indicate that maintenance of PCP signaling is posterior cell dependent. (3) ECM components mediate the interaction between $\mathrm{PCP}$ and $\mathrm{AB}$ polarities. Disruption of PCP signaling causes the mislocation of ECM component at the cell-cell interface (Veeman et al., 2007), suggesting that localization of basal ECM is PCP dependent. In addition, in zebrafish and Xenopus, disruption of the function of notochord sheath protein components, such as laminin or collagen, leads to abnormal notochord and vacuole morphogenesis (Parsons et al., 2002; Pagnon-Minot et al., 2008; Buisson et al., 2014; Manninen, 2015).

\section{POLARIZED ECM SECRETION IN THE NOTOCHORD}

Notochord tissue in different species has a few common features, such as the notochord sheath (closed tube component of the ECM) and the lumen cavity formed by distinct ways. In Ciona, notochord cells form an extracellular lumen through MET, surrounded by a single layer of notochord cells and an ECM notochord sheath (Dong et al., 2009). In zebrafish, some notochord cells form a layer of outer notochord sheath cells along the notochord sheath while other cells move to an inner cell core, and vacuoles are formed within each cell (Ellis et al., 2013a,b; Corallo et al., 2015). In amniotes, such as chicken and mice, vacuolated notochord cells are surrounded by an acellular sheath (Choi et al., 2008; Ward et al., 2018).

Notochord sheath formation is oriented by secretion of the polarized basal surface ECM. In Xenopus, the chordamesoblast forms radial polarization induced by surrounding tissues and then secretes fibronectin at the basement surface, forming a fibronectin layer between the mesoderm and surrounding tissues. Disruption of the $\mathrm{Pk}$ and/or Stbm/Fz function leads to failure of basal ECM secretion (Goto et al., 2005), indicating that the Wnt/PCP signaling pathway is essential for basal ECM secretion. Similarly, failure of laminin (polarized ECM component) secretion is found in Pk mutant Ciona embryos (Veeman et al., 2007; Veeman and McDonald, 2016), indicating that a conserved regulatory mechanism underlies basal ECM secretion. In addition to the Wnt/PCP signaling pathway, other signaling pathways, such as hedgehog, might also be involved in notochord sheath formation (Choi and Harfe, 2011).

In addition to basal ECM secretion, apical ECM secretion is also found sometimes (Mizotani et al., 2018; Bhattachan et al., 2020), which forms extracellular pocket lumens. During this process, notochord polarity moves from $\mathrm{PCP}$ to $\mathrm{AB}$ polarity (Dong et al., 2009). Both PCP an AB polarities and basal vesical trafficking are required for apical ECM secretion (Mizotani et al., 2018).

\section{CYTOSKELETON POLARITIES IN Ciona NOTOCHORD MORPHOGENESIS}

The cytoskeleton, including the microtubule, actin filaments, and intermediate filaments, is a group of fibriform intracellular proteins that form a skeleton network to maintain the cell shape and movement (Pegoraro et al., 2017). Cytoskeleton polarity, such as asymmetry distribution; oriented arrangement; and polar crosslinking, cooperation, disassociation, and rearrangement, plays an important role in cell asymmetry structures and behaviors. A polarized cytoskeleton is the main cause of an unbalanced bioforce. In contrast, a cytoskeleton is highly dynamic. It can be sensitively regulated and under the control of cell polarity (Raman et al., 2018). Therefore, cytoskeleton polarity is an important pathway to externalize intracellular polar signaling.

Cytoskeleton polarity plays an important role in diverse notochord morphogenesis, including the ascidian notochord. At 


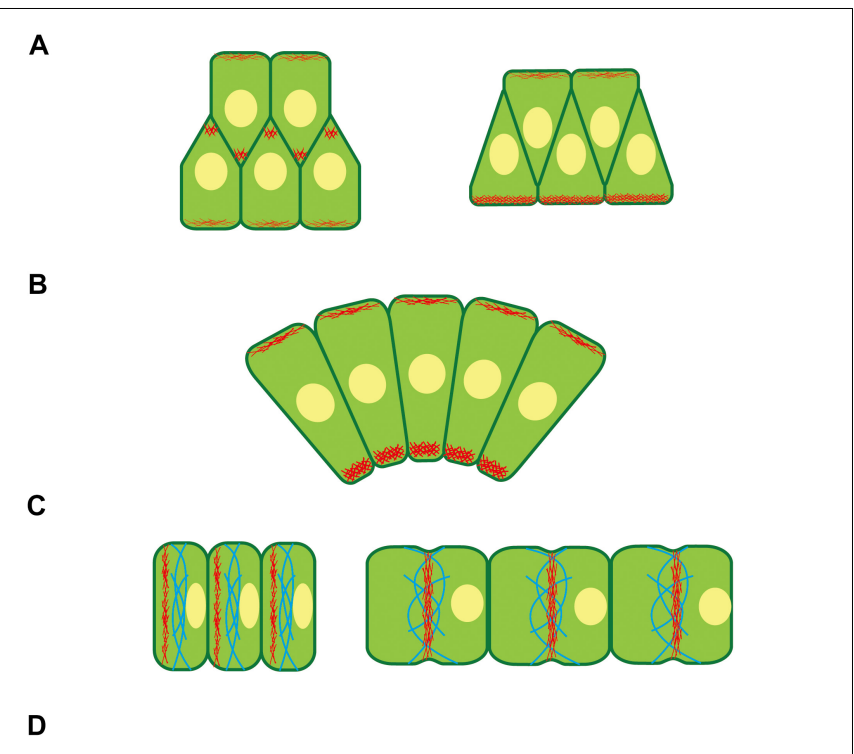

D
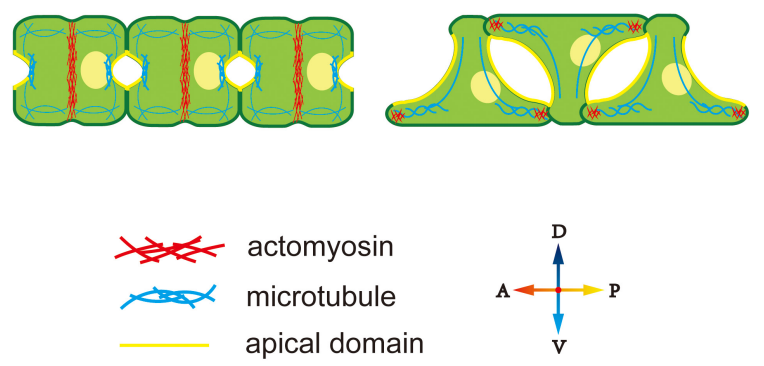

FIGURE 2 | Cytoskeleton polarity during notochord development in Ciona. (A) F-actin accumulates at the lamellipodium tip, providing migrating forces for cell intercalation. (B) Ventrally accumulated actomyosin contractility provides unbalanced force to drive notochord bending. (C) An actomyosin contractile ring forms at the anterior edge and moves to the equator of notochord cells. Actomyosin ring contraction elongates notochord cells. Microtubules are perpendicular to the AP axis within notochord cells. (D) During lumen expansion, microtubules accumulate at the apical domain, and along with bidirectional migration, they rotate $90^{\circ}$ and form oriented bundles toward the leading edges of tractive lamellipodia-like protrusions. An actomyosin contractile ring also exists during lumen expansion and then disappears. At bidirectional migration, F-actin moves to the tip of lamellipodia-like protrusions.

the early stage of notochord development in Ciona notochord, cytoskeleton distribution is regulated by the PCP signaling pathway (Sasakura and Makabe, 2001; Niwano et al., 2009), which induces accumulation of midline-oriented F-actin at the membrane cortex, forming cell protrusions (Figure 2A) (Munro and Odell, 2002). These protrusions generate a mechanical force to drive cell extension and migration across the surfaces of neighbor notochord cells toward the midline. Disruption of the function of PCP components, causes notochord cells to move in random directions instead of toward the midline (Jiang et al., 2005).

During CE, the notochord bends ventrally to adapt to the limited space inside the chorion. During this process, both actin and myosin II present an obviously ventral-polarized distribution in notochord cells. The dorsal-ventral (DV) polarity of the cytoskeleton in the notochord is observed during cell intercalation (Figure 2A). At this stage, notochord cells are arranged in two rows, while, actomyosin accumulates at the ventral cortex of the ventral row, that is, the ventral boundary of notochord tissue (Figure 2A, right panel). The polarized actomyosin generates a mechanical force that, along with the faster dorsal epidermis proliferation, drives notochord bending, therefore, embryonic tail bends. The most obvious actomyosin polarity appears at the anterior part of the notochord, where the bending angle is largest (Figure 2B). The cytoskeleton DV polarity can only be found in the ventral row during cell intercalation. However, when notochord cells in the dorsal row migrate across the ventral row, they immediately get DV polarity, indicating that the dorsal and ventral rows of notochord cell populations build DV polarity at different time and that establishment of cytoskeleton DV polarity in notochord cells depends on whether the cells are in contact with the ventral boundary of notochord tissue. The signaling pathway that controls DV-polarized cytoskeleton is unclear. The ECM protein laminin localizes dorsally (Veeman et al., 2007), while the apical cell polarity molecule aPKC localized ventrally (Oda-Ishii et al., 2010), which might provide a polarizing signal for polarized actomyosin enrichment. The aPKC has been demonstrated to phosphorylate Lgl to inhibit its interaction with myosin II (Betschinger et al., 2003), providing a cue for further study on the mechanism how D-V polarity influences cell behavior.

After CE, F-actin, and non-muscle myosin present as an actomyosin contractile ring perpendicular to the AP axis to drive notochord cell elongation (Dong et al., 2011; Sehring et al., 2014; Lu et al., 2019). The actomyosin contractile ring first appears at the anterior edge of the notochord and then migrates toward the equator (Figure 2C). Positioning of the actomyosin contractile ring is regulated by a "tug-of-war" mechanism between PCP signaling and actomyosin contractility. Inhibition of contractility causes the return of contractile ring to the anterior side, while disruption of both PCP and contractility causes random positioning of contractile ring, indicating that the PCP determines the initial ring position and contractility drives the ring to move to the equator (Sehring et al., 2015). More actomyosin accumulates at the anterior edge compared to the posterior membrane (Newman-Smith et al., 2015). The anterior actomyosin cytoskeleton and core PCP component mutually colocalize at the anterior membrane domain. They interact and maintain the stable 1D PCP along the AP axis. The cytoskeleton is controlled by cell polarity; conversely, cytoskeleton polarity regulates the localization of key polarity-signaling molecules.

After notochord cell elongation, an extracellular lumen forms and then expands between adjacent notochord cells (Dong et al., 2009). This process begins with PCP-AB polarity transition (Denker et al., 2013). The mechanism underlying polarity transition is unclear, but cytoskeleton polarity is likely involved. AB polarity formation in Drosophila depends on the F-actin cytoskeleton (Schmidt et al., 2018; Sokac and Wieschaus, 2008a,b). In notochord cells in Ciona, a polarized microtubule network is seen toward the apical membrane, which might signal $\mathrm{AB}$ polarity formation (Figure 2D). Cortical actin and ERM are found to be essential for lumen formation (Dong et al., 2011). 
This cytoskeleton network might function as a vesicle-trafficking track to secret the ECM into the extracellular lumen (Mizotani et al., 2018; Bhattachan et al., 2020).

When the lumen expands to a specific volume, notochord cells begin to migrate bidirectionally and flatten (Figure 2D). Lamellipodia-like protrusions, whose formation depends on the F-actin network, provide the mechanical force for migration (Dong et al., 2011). Disruption of F-actin polymerization causes the protrusions to disappear and notochord cells to lose migration ability. At the stage of protrusion formation, the microtubule cytoskeleton rotates $90^{\circ}$ and forms oriented bundles toward the leading edges of tractive lamellipodia (Dong et al., 2011). Inhibiting microtubule assembling leads to abnormal the location and number of cell protrusions, indicating that polarized microtubule provides directional information for notochord cell migration.

\section{POLARITY SIGNALING REGULATES MECHANICAL PROPERTIES OF THE NOTOCHORD TISSUE}

Development is a coordinated multi-tissue-reshaping and movement process. It is also a process of biomechanical force generation, conduction, and release. During development, different tissues have distinct mechanical properties, leading to different reshaping and migratory patterns. A tissue, as a type of specific biomaterial, has autokinetic movement ability, which is a characteristic of life. Therefore a tissue's mechanical properties include not only a shape-changing ability under force, such as bending, stretching, compressing, and twisting, which are described by materials science, but also a regulatory ability to magnify, locate, and orientate bioforces (Mammoto and Ingber, 2010). The tissue's mechanical properties are determined by diverse bioforces generated by subcellular or supracellular structures, such as the cytoskeleton, nuclei, ECM, and cell junctions (Ingber, 2006). Polarity, as dynamic signaling, drives a change in a tissue's mechanical properties by regulating these subcellular or supracellular structures to match embryo developmental processes. Therefore, polarity can serve as a bridge connecting signaling regulation and tissue mechanical properties and coordinate organogenesis during embryo development.

The notochord, as a center pillar-like structure is the main support organ of chordate embryo development (Corallo et al., 2015). In addition, the notochord is a typical polarityinduced organ, and many polarity regulatory pathways are involved in notochord formation. Therefore, the notochord is an important model to study how polarity regulates a tissue's mechanical properties.

At the intercellular level, polarity regulates not match with the properties cell's mechanical properties by affecting the cytoskeleton, nuclei position, or oriented vesicle transport. The cytoskeleton, as an intracellular support structure, is the main cause of an unbalanced bioforce. A polarity signal, such as a PCP signal, regulates the Rho signal pathway to affect cytoskeleton distribution (Nishimura et al., 2012), while cytoskeleton localization and orientation creates cells' mechanical properties (Hefele et al., 2004; Shin et al., 2007).
As described earlier, F-actin accumulates at the tip of the lamellipodium to force cell migration toward the midline during cell intercalation (Figure 3Ai). In notochord cells in Ciona, the ventrally accumulated actomyosin contracts to generate a force that bends the notochord (Figure 3Aii). Actomyosin contractile rings localize at the middle of the cells to drive cell elongation (Figure 3Aiii). Therefore, cytoskeleton localization affects cells' mechanical properties, while polarized oriented microtubule bundles help lumen expansion and protrusion formation, indicating how cytoskeleton orientation changes cells' mechanical properties (Dong et al., 2011).

The asymmetric position of nuclei also regulates cells' mechanical properties (Calero-Cuenca et al., 2018). During notochord cell elongation in Ciona embryos, PCP signaling drives the posterior location of the nuclei in notochord cells (Figure 3Aiii) (Jiang et al., 2005; Kourakis et al., 2014). However, the underlying mechanism is still unclear and might be related to the notochords' mechanical properties. In amoeboid migration of immune or lobopodial cells, the nucleus' position affects mechanical properties, such as strength and migration ability (Petrie et al., 2014; Salvermoser et al., 2018; Renkawitz et al., 2019).

In addition, polarity controls polarized membrane vesicle trafficking, which in turn affects cells' mechanical properties. During notochord lumen expansion in Ciona, vesicles are transported toward the apical domain to supply the apical membrane and release the membrane tension from continuous lumen expansion (Figure 3Biv) (Mizotani et al., 2018; Bhattachan et al., 2020).

At the supracellular level, polarity can regulate oriented ECM secretion, which directly affects cells' mechanical properties by forming a crosslinked network to provide enough strength and elasticity or indirectly regulates by contributing to lumen formation (Loganathan et al., 2020). In the notochord in Ciona, basal ECM secretion forms a basal lamina-like notochord sheath (Figure 3Bv) (Wei et al., 2017). In addition, some of the ECM has high hydrophilicity, significantly increasing osmotic pressure (Lu et al., 2006). Polarity signaling controls the fixed-point release of the ECM to control osmotic pressure variation in specific areas to influence extracellular liquid flow (Figure 3Cvi). There is a lot of ECM in the notochord lumen in Ciona, which is secreted by the apical membrane to increase osmotic pressure. Apical lumen expansion together with the basal notochord sheath, forms a fiber-wound cylinder hydrostatic skeleton. Since it is difficult to compress the liquid limen, this structure impacts a higher strength to the notochord than the limit of ECM's and notochord cells' mechanical properties (Adams et al., 1990; Stemple, 2005; Yasuoka, 2020). In addition, the flexibility of this fiber-wound cylinder structure is higher compared to cell reshaping and ECM remodeling. Therefore, it facilitates dynamic regulation of the notochord to adapt to faster embryo development. In Ciona, the notochord forms a connected single lumen. In contract, in zebrafish, the notochord forms multiple, discontinuous intracellular vacuoles (Ellis et al., 2013a,b; Corallo et al., 2015). The high connectivity of the lumen might be beneficial by dispersing the strength through lumen matrix flow, which impacts to the notochord greater impact resistance. 


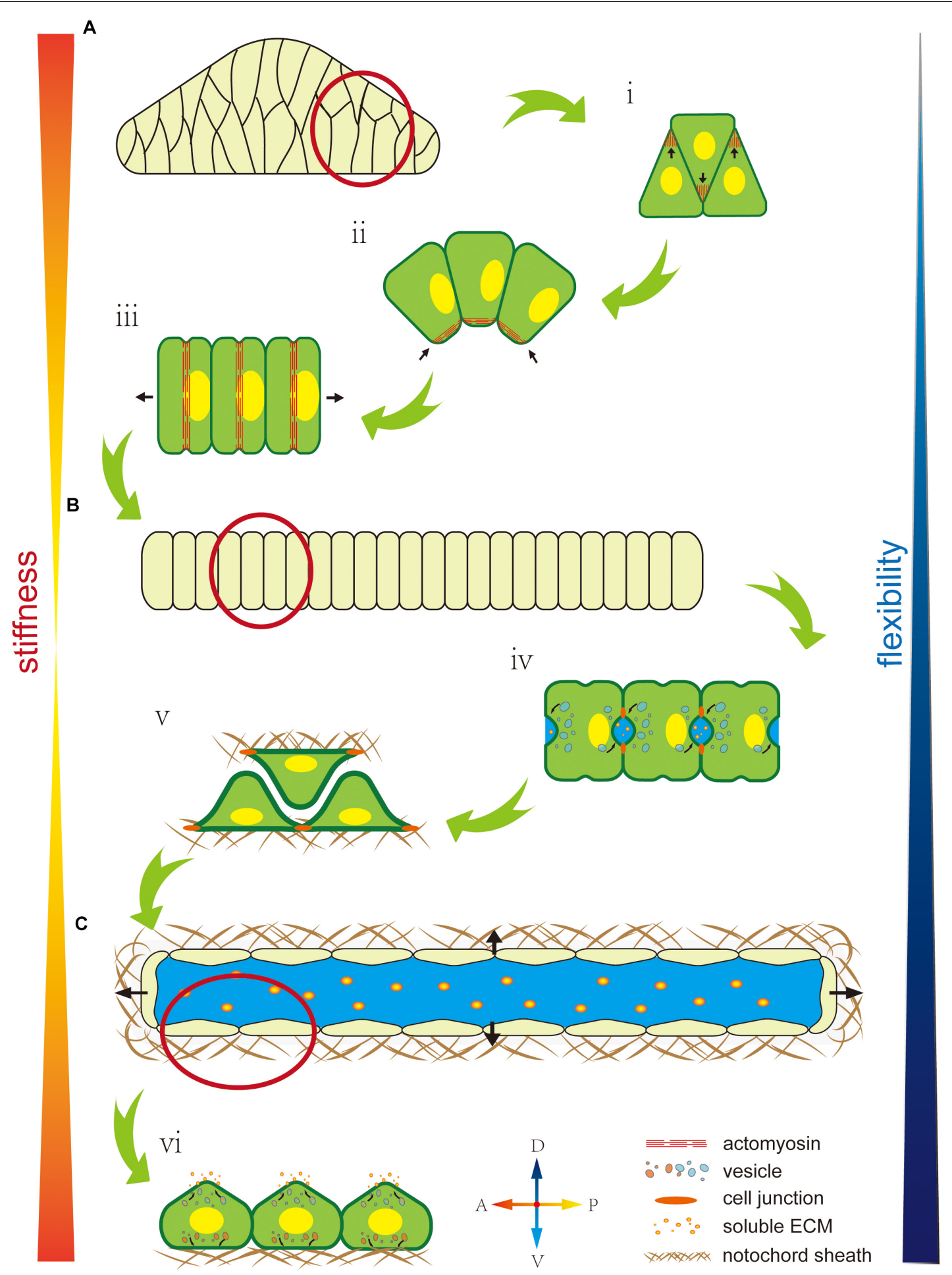

FIGURE 3 | Mechanical properties of the notochord are regulated by cell polarity. (A) The early notochord is a short, thick, stiff, rod-like structure made up of a multiline of cells. (i) Polarized F-actin drives cells to migrate and intercalate to form a single line. (ii) Ventrally polarized actomyosin drives notochord bending toward the ventral side. (iii) An equator-localized contractile ring drives notochord cell elongation. (B) The notochord forms as a long, thin, single cell-line cord. Its stiffness is low but flexibility is high, which facilitates rapid embryonic development in the narrow space inside the chorion. (iv) AB polarity is built during extracellular lumen formation. (v) Notochord cells migrate bidirectionally along the notochord sheath. (vi) Notochord cells form an endothelial-like single layer. (C) A single lumen-filled notochord tube increases tissue stiffness and flexibility, which enables the swimming of Ciona larva in seawater. 
However, this structure cannot control the shape locally. The evolutionary trend from a single lumen to multiple disconnected vacuoles might compromise the notochord stiffness but impart tissue flexibility, which makes the notochord adapt to the more complex developmental processes in vertebrates.

\section{SUMMARY AND PERSPECTIVE}

Ciona notochord is composing of 40 cells with diverse polarities phenomena during development, being an ideal simple model for polarity study. Besides, the Ciona genome is single-copy, which benefits to elucidate the molecular mechanism for polarity establishment and maintenance. Because of the specifically evolutionary position in animal phylogenetic taxa, the knowledge achieved from Ciona notochord polarity can be used for reference toward both invertebrate and vertebrate.

Although the Ciona notochord model is simple for polarity study, there still remains several challenge questions need to be answered in the future. For example, what is the signaling contributing to the establishment of the initial $\mathrm{AB}$ polarity? Who mediates the interaction and transition between PCP and $\mathrm{AB}$ polarity? How do polarities coordinate the concerted and coherent development in multiple tissues?

Par/aPKC complex contributes to the establishment of $\mathrm{AB}$ polarity in notochord cell. However, the signaling for the establishment of initial $\mathrm{AB}$ polarity is still unknown. It has been indicated that Rho signaling mediated centrosome and microtubule play an important role in the initial localization of the key component proteins of $\mathrm{AB}$ polarity. Further study to reveal the controlling signals for the localization of Par/aPKC complex in Ciona notochord cells will provide cues to understand how $\mathrm{AB}$ polarity is initially established in a cell.

The crosstalk between PCP and AB polarity is also worth further exploring to learn about how the different polarity systems are coordinated. Several ways including the interaction between PCP and AB polarity components, cell-cell contact, and cell-basement membrane contact are candidate pathways to

\section{REFERENCES}

Adams, D. S., Keller, R., and Koehl, M. A. (1990). The mechanics of notochord elongation, straightening and stiffening in the embryo of Xenopus laevis. Development 110, 115-130.

Adler, P. N. (2012). The frizzled/stan pathway and planar cell polarity in the Drosophila wing. Curr. Top. Dev. Biol. 101, 1-31. doi: 10.1016/b978-0-12394592-1.00001-6

Assémat, E., Bazellières, E., Pallesi-Pocachard, E., Le Bivic, A., and MasseyHarroche, D. (2008). Polarity complex proteins. Biochim. Biophys. Acta 1778, 614-630.

Bagwell, J., Norman, J., Ellis, K., Peskin, B., Hwang, J., Ge, X., et al. (2020). Notochord vacuoles absorb compressive bone growth during zebrafish spine formation. eLife 9:e51221.

Balser, E. J., and Ruppert, E. E. (1990). Structure, ultrastructure, and function of the preoral heart-kidney in Saccoglossus kowalevski (Hemichordata, Enteropneusta) including new data on the stomochord. Acta Zool. 71, 235-249. doi: 10.1111/j.1463-6395.1990.tb01082.x

Bénazéraf, B., and Pourquié, O. (2013). Formation and segmentation of the vertebrate body axis. Annu. Rev. Cell Dev. Biol. 29, 1-26. doi: 10.1146/annurevcellbio-101011-155703 mediate such crosstalk between PCP and AB polarity. However, the molecular mechanisms underlying these possibilities are unknown. Investigation in a structurally simple and single gene copy notochord system will benefit to answer the question.

Polarity plays an important role in coordination of multiple tissue development by controlling tissue mechanical property. For example, when flat cell sheets bundle to form a biological tube, polarity regulates an asymmetry cell wedge or drives cell directional intercalate, leading to the bending movement through the mechanical property change in different parts of the cell flat sheet (Nielsen et al., 2020). Compared with the anatomically complex of vertebrate, Ciona embryonic tail is structurally simple, providing an excellent model to understand how polarity signaling coordinates the multiple tissue development.

\section{AUTHOR CONTRIBUTIONS}

$\mathrm{BD}$ and HP conceived and wrote the manuscript. HP prepared the figures. RQ provided critical editing and input. All authors contributed to the article and approved the submitted version.

\section{FUNDING}

BD was supported by the National Key Research and Development Program of China (2019YFE0190900), the National Natural Science Foundation of China (31771649), the Marine S\&T Fund of Shandong Province for Pilot National Laboratory for Marine Science and Technology (Qingdao) (2018SDKJ0302-1).

\section{ACKNOWLEDGMENTS}

We thank members of the Dong Laboratory, including Zhiyi Lv, Xiuxia Yang, and Wenjie Shi for their critical reading and invaluable comments on the manuscript.

Betschinger, J., Mechtler, K., and Knoblich, J. A. (2003). The Par complex directs asymmetric cell division by phosphorylating the cytoskeletal protein Lgl. Nature 422, 326-330. doi: 10.1038/nature01486

Bhattachan, P., Rae, J., Yu, H., Jung, W., Wei, J., Parton, R. G., et al. (2020). Ascidian caveolin induces membrane curvature and protects tissue integrity and morphology during embryogenesis. FASEB J. 34, 1345-1361. doi: 10.1096/ fj.201901281r

Bisgrove, B. W., Essner, J. J., and Yost, H. J. (2000). Multiple pathways in the midline regulate concordant brain, heart and gut left-right asymmetry. Development 127, 3567-3579.

Brownlee, C., and Bouget, F. (1998). Polarity determination in fucus: from zygote to multicellular embryo. Semin. Cell Dev. Biol. 9, 179-185. doi: 10.1006/scdb. 1997.0212

Brunet, T., Lauri, A., and Arendt, D. (2015). Did the notochord evolve from an ancient axial muscle? The axochord hypothesis. Bioessays 37, 836-850. doi: 10.1002/bies.201500027

Buisson, N., Sirour, C., Moreau, N., Denker, E., Le Bouffant, R., Goullancourt, A., et al. (2014). An adhesome comprising laminin, dystroglycan and myosin IIA is required during notochord development in Xenopus laevis. Development 141, 4569-4579. doi: 10.1242/dev.11 6103 
Calero-Cuenca, F. J., Janota, C. S., and Gomes, E. R. (2018). Dealing with the nucleus during cell migration. Curr. Opin. Cell Biol. 50, 35-41. doi: 10.1016/ j.ceb.2018.01.014

Choi, K., Cohn, M. J., and Harfe, B. D. (2008). Identification of nucleus pulposus precursor cells and notochordal remnants in the mouse: implications for disk degeneration and chordoma formation. Dev. Dyn. 237, 3953-3958. doi: 10. 1002/dvdy.21805

Choi, K. S., and Harfe, B. D. (2011). Hedgehog signaling is required for formation of the notochord sheath and patterning of nuclei pulposi within the intervertebral discs. Proc. Natl. Acad. Sci. U.S.A. 108, 9484-9489. doi: 10.1073/pnas. 1007566108

Cloney, R. A. (1982). Ascidian larvae and the events of metamorphosis. Am. Zool. 22, 817-826. doi: 10.1093/icb/22.4.817

Corallo, D., Trapani, V., and Bonaldo, P. (2015). The notochord: structure and functions. Cell. Mol. Life Sci. 72, 2989-3008. doi: 10.1007/s00018-015-1897-z

Deng, W., Nies, F., Feuer, A., Bocina, I., Oliver, D., and Jiang, D. (2013). Anion translocation through an Slc26 transporter mediates lumen expansion during tubulogenesis. Proc. Natl. Acad. Sci. U.S.A. 110, 14972-14977. doi: 10.1073/ pnas. 1220884110

Denker, E., and Jiang, D. (2012). Ciona intestinalis notochord as a new model to investigate the cellular and molecular mechanisms of tubulogenesis. Semin. Cell Dev. Biol. 23, 308-319. doi: 10.1016/j.semcdb.2012.03.004

Denker, E., Bocina, I., and Jiang, D. (2013). Tubulogenesis in a simple cell cord requires the formation of $\mathrm{Bi}$-apical cells through two discrete par domains. Development 140, 2985-2996. doi: 10.1242/dev.092387

Dhonukshe, P. (2013). "Polarity, developmental," in Brenner's Encyclopedia of Genetics, 2nd Edn, eds S. Maloy and K. Hughes (Cambridge, MA: Academic Press), 378-382.

Djiane, A., Yogev, S., and Mlodzik, M. (2005). The apical determinants aPKC and dPatj regulate frizzled-dependent planar cell polarity in the Drosophila eye. Cell 121, 621-631. doi: 10.1016/j.cell.2005.03.014

Dong, B., Horie, T., Denker, E., Kusakabe, T., Tsuda, M., Smith, W. C., et al. (2009). Tube formation by complex cellular processes in Ciona intestinalis notochord. Dev. Biol. 330, 237-249. doi: 10.1016/j.ydbio.2009.03.015

Dong, B., Deng, W., and Jiang, D. (2011). Distinct cytoskeleton populations and extensive crosstalk control Ciona notochord tubulogenesis. Development 138, 1631-1641. doi: 10.1242/dev.057208

Ellis, K., Bagwell, J., and Bagnat, M. (2013a). Notochord vacuoles are lysosomerelated organelles that function in axis and spine morphogenesis. J. Cell Biol. 200, 667-679. doi: 10.1083/jcb.201212095

Ellis, K., Hoffman, B. D., and Bagnat, M. (2013b). The vacuole within: how cellular organization dictates notochord function. Bioarchitecture 3, 64-68. doi: 10 . 4161/bioa.25503

Eom, D. S., Amarnath, S., Fogel, J. L., and Agarwala, S. (2011). Bone morphogenetic proteins regulate neural tube closure by interacting with the apicobasal polarity pathway. Development 138, 3179-3188. doi: 10.1242/dev.058602

Feldman, J. L., and Priess, J. R. (2012). A role for the centrosome and PAR-3 in the hand-off of MTOC function during epithelial polarization. Curr. Biol. 22, 575-582. doi: 10.1016/j.cub.2012.02.044

Feng, Y., Li, J., and Xu, A. (2016). "Amphioxus as a model for understanding the evolution of vertebrates," in Amphioxus Immunity, ed. A. Xu (Cambridge, MA: Academic Press), 1-13. doi: 10.1016/b978-0-12-849903-0.00001-4

Goto, T., Davidson, L., Asashima, M., and Keller, R. (2005). Planar cell polarity genes regulate polarized extracellular matrix deposition during frog gastrulation. Curr. Biol. 15, 787-793. doi: 10.1016/j.cub.2005.03.040

Grimes, D. T. (2019). Making and breaking symmetry in development, growth and disease. Development 146:dev170985. doi: 10.1242/dev.170985

Hashimoto, M., and Hamada, H. (2010). Translation of anterior-posterior polarity into left-right polarity in the mouse embryo. Curr. Opin. Genet. Dev. 20, 433-437. doi: 10.1016/j.gde.2010.04.002

Hefele, J. A., Unterseher, F., Giehl, K., Schambony, A., Wedlich, D., and De Robertis, E. M. (2004). Paraxial protocadherin coordinates cell polarity during convergent extension via Rho A and JNK. EMBO J. 23, 3259-3269. doi: 10. 1038/sj.emboj.7600332

Hermann, G. J., Leung, B., and Priess, J. R. (2000). Left-right asymmetry in C. elegans intestine organogenesis involves a LIN-12/Notch signaling pathway. Development 127, 3429-3440.

Holló, G. (2015). A new paradigm for animal symmetry. Interface Focus 5, 1-10.
Hu, G., Li, G., Wang, H., and Wang, Y. (2017). Hh gene participates in the leftright asymmetry development of amphioxus by controlling Cer expression. Development 144, 4694-4703. doi: 10.1242/dev.157172

Ingber, D. E. (2006). Cellular mechanotransduction: putting all the pieces together again. FASEB J. 20, 811-827. doi: 10.1096/fj.05-5424rev

Jiang, D., Munro, E. M., and Smith, W. C. (2005). Ascidian prickle regulates both mediolateral and anterior-posterior cell polarity of notochord cells. Curr. Biol. 15, 79-85. doi: 10.1016/j.cub.2004.12.041

Kato, Y. (2011). The multiple roles of Notch signaling during left-right patterning. Cell. Mol. Life Sci. 68, 2555-2567. doi: 10.1007/s00018-011-0695-5

Keller, R. (2006). Mechanisms of elongation in embryogenesis. Development 133, 2291-2302. doi: 10.1242/dev.02406

Kourakis, M. J., Reeves, W., Newman-Smith, E., Maury, B., Abdul-Wajid, S., and Smith, W. C. (2014). A one-dimensional model of PCP signaling: polarized cell behavior in the notochord of the ascidian Ciona. Dev. Biol. 395, 120-130. doi: 10.1016/j.ydbio.2014.08.023

Lauri, A., Brunet, T., Handberg-Thorsager, M., Fischer, A. H. L., Simakov, O., Steinmetz, P. R. H., et al. (2014). Development of the annelid axochord: insights into notochord evolution. Science 345, 1365-1368. doi: 10.1126/ science. 1253396

Loganathan, R., Little, C. D., and Rongish, B. J. (2020). Extracellular matrix dynamics in tubulogenesis. Cell. Signal. 72:109619. doi: 10.1016/j.cellsig.2020. 109619

Lu, Q., Bhattachan, P., and Dong, B. (2019). Ascidian notochord elongation. Dev. Biol. 448, 147-153. doi: 10.1016/j.ydbio.2018.11.009

Lu, Y., Parker, K. H., and Wang, W. (2006). Effects of osmotic pressure in the extracellular matrix on tissue deformation. Philos. Trans. R. Soc. A Math. Phys. Eng. Sci. 364, 1407-1422. doi: 10.1098/rsta.2006.1778

Lv, Z., Lu, Q., and Dong, B. (2019). Morphogenesis: a focus on marine invertebrates. Mar. Life Sci. Technol. 1, 28-40. doi: 10.1007/s42995-01900016-z

Mammoto, T., and Ingber, D. E. (2010). Mechanical control of tissue and organ development. Development 137, 1407-1420. doi: 10.1242/dev.024166

Manninen, A. (2015). Epithelial polarity - generating and integrating signals from the ECM with integrins. Exp. Cell Res. 334, 337-349. doi: 10.1016/j.yexcr.2015. 01.003

Marsden, M., and DeSimone, D. W. (2003). Integrin-ECM interactions regulate cadherin-dependent cell adhesion and are required for convergent extension in Xenopus. Curr. Biol. 13, 1182-1191. doi: 10.1016/s0960-9822(03)00433-0

Matsunobu, S., and Sasakura, Y. (2015). Time course for tail regression during metamorphosis of the ascidian Ciona intestinalis. Dev. Biol. 405, 71-81. doi: 10.1016/j.ydbio.2015.06.016

McCann, M. R., Tamplin, O. J., Rossant, J., and Seguin, C. A. (2011). Tracing notochord-derived cells using a Noto-cre mouse: implications for intervertebral disc development. Dis. Models Mech. 5, 73-82. doi: 10.1242/dmm.008128

Mizotani, Y., Suzuki, M., Hotta, K., Watanabe, H., Shiba, K., Inaba, K., et al. (2018). 14-3-3ea directs the pulsatile transport of basal factors toward the apical domain for lumen growth in tubulogenesis. Proc. Natl. Acad. Sci. U.S.A. 115, E8873-E8881.

Mlodzik, M. (2002). "Tissue polarity in the retina," in Results and Problems in Cell Differentiation, Vol. 37, ed. K. Moses (Berlin: Springer), 89-106. doi: 10.1007/ 978-3-540-45398-7_7

Munro, E. M., and Odell, G. M. (2002). Polarized basolateral cell motility underlies invagination and convergent extension of the ascidian notochord. Development 129, 13-24.

Nance, J. (2014). Getting to know your neighbor: cell polarization in early embryos. J. Cell Biol. 206, 823-832. doi: 10.1083/jcb.201407064

Newman-Smith, E., Kourakis, M. J., Reeves, W., Veeman, M., and Smith, W. C. (2015). Reciprocal and dynamic polarization of planar cell polarity core components and myosin. eLife 4:e5361.

Nielsen, B. F., Nissen, S. B., Sneppen, K., Mathiesen, J., and Trusina, A. (2020). Model to link cell shape and polarity with organogenesis. iScience 23:100830. doi: $10.1016 /$ j.isci.2020.100830

Ninomiya, H. (2004). Antero-posterior tissue polarity links mesoderm convergent extension to axial patterning. Nature 430, 364-367. doi: 10.1038/nature02620

Nishimura, T., Honda, H., and Takeichi, M. (2012). Planar cell polarity links axes of spatial dynamics in neural-tube closure. Cell 149, 1084-1097. doi: 10.1016/j. cell.2012.04.021 
Niwano, T., Takatori, N., Kumano, G., and Nishida, H. (2009). Wnt5 is required for notochord cell intercalation in the ascidian Halocynthia roretzi. Biol. Cell 101, 645-659. doi: 10.1042/bc20090042

Oda-Ishii, I., Ishii, Y., and Mikawa, T. (2010). Eph regulates dorsoventral asymmetry of the notochord plate and convergent extension-mediated notochord formation. PLoS One 5:e13689. doi: 10.1371/journal.pone. 0013689

Ota, K. G., and Kuratani, S. (2007). Cyclostome embryology and early evolutionary history of vertebrates. Integr. Comp. Biol. 47, 329-337. doi: 10.1093/icb/ icm022

Pagnon-Minot, A., Malbouyres, M., Haftek-Terreau, Z., Kim, H. R., Sasaki, T., Thisse, C., et al. (2008). Collagen XV, a novel factor in zebrafish notochord differentiation and muscle development. Dev. Biol. 316, 21-35. doi: 10.1016/ j.ydbio.2007.12.033

Parsons, M. J., Pollard, S. M., Saúde, L., Feldman, B., Coutinho, P., Elizabeth, M. A. H., et al. (2002). Zebrafish mutants identify an essential role for laminins in notochord formation. Development 129, 3137-3146.

Pegoraro, A. F., Janmey, P., and Weitz, D. A. (2017). Mechanical properties of the cytoskeleton and cells. Cold Spring Harb. Perspect. Biol. 9:a022038. doi: 10.1101/cshperspect.a022038

Petrie, R. J., Koo, H., and Yamada, K. M. (2014). Generation of compartmentalized pressure by a nuclear piston governs cell motility in a 3D matrix. Science 345, 1062-1065. doi: 10.1126/science. 1256965

Pickett, M. A., Naturale, V. F., and Feldman, J. L. (2019). A polarizing issue: diversity in the mechanisms underlying apico-basolateral polarization in vivo. Annu. Rev. Cell Dev. Biol. 35, 285-308. doi: 10.1146/annurev-cellbio-100818125134

Raman, R., Pinto, C. S., and Sonawane, M. (2018). Polarized organization of the cytoskeleton: regulation by cell polarity proteins. J. Mol. Biol. 430, 3565-3584. doi: $10.1016 /$ j.jmb.2018.06.028

Raya, Á., and Belmonte, J. C. I. (2006). Left-right asymmetry in the vertebrate embryo: from early information to higher-level integration. Nat. Rev. Genet. 7, 283-293. doi: 10.1038/nrg1830

Renkawitz, J., Kopf, A., Stopp, J., de Vries, I., Driscoll, M. K., Merrin, J., et al. (2019). Nuclear positioning facilitates amoeboid migration along the path of least resistance. Nature 568, 546-550. doi: 10.1038/s41586-0191087-5

Salvermoser, M., Begandt, D., Alon, R., and Walzog, B. (2018). Nuclear deformation during neutrophil migration at sites of inflammation. Front. Immunol. 9:2680. doi: 10.3389/fimmu.2018.02680

Sasakura, Y., and Makabe, K. W. (2001). Ascidian Wnt-5 gene is involved in the morphogenetic movement of notochord cells. Dev. Growth Differ. 43, 573-582. doi: 10.1046/j.1440-169x.2001.00602.x

Schmidt, A., Lv, Z., and Großhans, J. (2018). ELMO and sponge specify subapical restriction of canoe and formation of the subapical domain in early Drosophila embryos. Development 145:dev157909. doi: 10.1242/dev.157909

Sehring, I. M., Dong, B., Denker, E., Bhattachan, P., Deng, W., Mathiesen, B. T., et al. (2014). An equatorial contractile mechanism drives cell elongation but not cell division. PLoS Biol. 12:e1001781. doi: 10.1371/journal.pbio. 1001781

Sehring, I. M., Recho, P., Denker, E., Kourakis, M., Mathiesen, B., Hannezo, E., et al. (2015). Assembly and positioning of actomyosin rings by contractility and planar cell polarity. eLife 4:e09206.

Shin, J. H., Tam, B. K., Brau, R. R., Lang, M. J., Mahadevan, L., and Matsudaira, P. (2007). Force of an actin spring. Biophys. J. 92, 3729-3733. doi: 10.1529/ biophysj.106.099994

Smith, W. C. (2018). Cellular processes of notochord formation. Adv. Exp. Med. Biol. 1029, 165-177. doi: 10.1007/978-981-10-7545-2_15

Sokac, A. M., and Wieschaus, E. (2008a). Local actin-dependent endocytosis is zygotically controlled to initiate Drosophila cellularization. Dev. Cell 14, 775-786. doi: 10.1016/j.devcel.2008.02.014
Sokac, A. M., and Wieschaus, E. (2008b). Zygotically controlled F-actin establishes cortical compartments to stabilize furrows during Drosophila cellularization. J. Cell Sci. 121, 1815-1824. doi: 10.1242/jcs.025171

Stemple, D. L. (2005). Structure and function of the notochord: an essential organ for chordate development. Development 132, 2503-2512. doi: 10.1242/dev. 01812

Suzuki, A., Yamanaka, T., Hirose, T., Manabe, N., Mizuno, K., Shimizu, M., et al. (2001). Atypical protein kinase $C$ is involved in the evolutionarily conserved par protein complex and plays a critical role in establishing epithelia-specific junctional structures. J. Cell Biol. 152, 1183-1196. doi: 10.1083/jcb.152.6.1183

Turing, A. M. (1952). The chemical basis of morphogenesis. Philos. Trans. R. Soc. Lond. B 237, 37-72.

Ukita, K., Hirahara, S., Oshima, N., Imuta, Y., Yoshimoto, A., Jang, C., et al. (2009). Wnt signaling maintains the notochord fate for progenitor cells and supports the posterior extension of the notochord. Mech. Dev. 126, 791-803. doi: 10.1016/j.mod.2009.08.003

Veeman, M. T., and McDonald, J. A. (2016). Dynamics of cell polarity in tissue morphogenesis: a comparative view from Drosophila and Ciona. F1000Res. 5:1084. doi: 10.12688/f1000research.8011.1

Veeman, M. T., Nakatani, Y., Hendrickson, C., Ericson, V., Lin, C., and Smith, W. C. (2007). Chongmague reveals an essential role for laminin-mediated boundary formation in chordate convergence and extension movements. Development 135, 33-41. doi: 10.1242/dev.010892

Ward, L., Pang, A. S. W., Evans, S. E., and Stern, C. D. (2018). The role of the notochord in amniote vertebral column segmentation. Dev. Biol. 439, 3-18. doi: 10.1016/j.ydbio.2018.04.005

Wedlich-Soldner, R., and Li, R. (2003). Spontaneous cell polarization: undermining determinism. Nat. Cell Biol. 5, 267-270. doi: 10.1038/ncb0403-267

Wei, J., Wang, G., Li, X., Ren, P., Yu, H., and Dong, B. (2017). Architectural delineation and molecular identification of extracellular matrix in ascidian embryos and larvae. Biol. Open 6, 1383-1390. doi: 10.1242/bio. 026336

Wen, W., and Zhang, M. (2018). Protein complex assemblies in epithelial cell polarity and asymmetric cell division. J. Mol. Biol. 430, 3504-3520. doi: 10. 1016/j.jmb.2017.09.013

Wolpert, L. (2013). Cell polarity. Philos. Trans. R. Soc. Lond. B Biol. Sci. 368:20130419.

Wopat, S., Bagwell, J., Sumigray, K. D., Dickson, A. L., Huitema, L. F. A., Poss, K. D., et al. (2018). Spine patterning is guided by segmentation of the notochord sheath. Cell Rep. 22, 2026-2038. doi: 10.1016/j.celrep.2018. 01.084

Wu, J., and Mlodzik, M. (2009). A quest for the mechanism regulating global planar cell polarity of tissues. Trends Cell Biol. 19, 295-305. doi: 10.1016/j.tcb.2009.04. 003

Yang, Y., and Mlodzik, M. (2015). Wnt-frizzled/planar cell polarity signaling: cellular orientation by facing the wind (Wnt). Annu. Rev. Cell Dev. Biol. 31, 623-646. doi: 10.1146/annurev-cellbio-100814-125315

Yasuoka, Y. (2020). Morphogenetic mechanisms forming the notochord rod: the turgor pressure-sheath strength model. Dev. Growth Differ. 62, 379-390. doi: $10.1111 /$ dgd. 12665

Conflict of Interest: The authors declare that the research was conducted in the absence of any commercial or financial relationships that could be construed as a potential conflict of interest.

Copyright (c) 2020 Peng, Qiao and Dong. This is an open-access article distributed under the terms of the Creative Commons Attribution License (CC BY). The use, distribution or reproduction in other forums is permitted, provided the original author(s) and the copyright owner(s) are credited and that the original publication in this journal is cited, in accordance with accepted academic practice. No use, distribution or reproduction is permitted which does not comply with these terms. 